Radiation-induced synergistic effects of athermal and thermal mechanisms on erosion and surface evolution of advanced electrode and condenser

Jean Paul Allain

Ahmed Hassanein

Tatiana Burtseva

Abdellatif Yacout

Zinetulla Insepov

Sarfraz Taj

Bryan J. Rice 


\title{
Radiation-induced synergistic effects of athermal and thermal mechanisms on erosion and surface evolution of advanced electrode and condenser optics materials
}

\author{
Jean P. Allain* ${ }^{1}$, Ahmed Hassanein ${ }^{1}$, Tatiana Burtseva ${ }^{1}$, Abdelatif Yacout ${ }^{1}$, \\ Zinetulla Insepov ${ }^{1}$, Sarfraz Taj ${ }^{3}$, Bryan J. Rice ${ }^{2}$ \\ Argonne National Laboratory, Argonne, IL, USA 60439 \\ Intel Corporation, Components Research, Hillsboro, OR, USA \\ University of Illinois at Urbana-Champaign, Urbana, IL, USA 61801
}

\begin{abstract}
In extreme ultraviolet lithography (EUVL) environments transient plasma dynamics dictate conditions for particle/surface interactions. A critical challenge facing EUVL development is optic component lifetime both in gasdischarge produced plasmas (GDPP) and laser-produced plasmas (LPP) devices. Optic components are exposed to impingent species, impurities (H,C,O,N) and debris leading to their degradation and consequently limiting $13.5 \mathrm{~nm}$ light reflection intensity. Experiments in the PRIME (Particles and Radiation Interaction with Matter Experiments) facility at the Argonne National Laboratory study the synergy between radiation-induced athermal and thermal mechanisms that influence the behavior of EUVL materials (electrodes and condenser optics) under irradiation conditions including: incident particle energy $(50 \mathrm{eV}-5 \mathrm{keV})$, angle-of-incidence (near-normal to oblique), incident flux $\left(10^{11}-10^{17}\right.$ ions $/ \mathrm{cm}^{2} / \mathrm{s}$ ), surface coatings (impurity: $\mathrm{C}, \mathrm{O}$ or capping layers: $\mathrm{Ru}, \mathrm{W}$ ), and surface temperature $\left(100-1000{ }^{\circ} \mathrm{C}\right)$. Results of electrode and optical component interaction with singly-charged inert gases (Xe) are presented. Critical issues under study include: radiation enhanced diffusion, radiation induced segregation, preferential sputtering, collisional mixing, surface segregation, surface amorphization, thermal diffusion and thermal spike evolution. Experiments in PRIME will be complemented with atomistic modeling to study how these mechanisms modify surfaces and how these mechanisms can work synergistically to introduce solutions to enhance component lifetime of electrode and condenser optic materials.
\end{abstract}

Keywords: diffusion, segregation, preferential sputtering, amorphization, condenser optics, collisional mixing

\section{INTRODUCTION}

One of the outstanding issues with GDPP (gas-discharge plasma produced) source devices for EUV lithography is lifetime of the $\mathrm{C} 1$ optics components. Lifetime is currently defined as the loss of EUV reflectivity of about $10 \%$ after operation with about $10^{11}$ shots. The fabrication characteristics and component properties of the Mo/Si multilayers are determining factors for the reflective intensities of the C1 optics mirrors in LPP (laser plasma produced) systems. Design of multilayers include optimum layer period as well as control of compressive vs tensile stresses. Optimization of these parameters as well as maintaining low surface roughness and using interface engineering ${ }^{1}$ results in increased $\mathrm{C} 1$ optics lifetime and greater mean reflective intensities for lithography. Additional issues include: interlayer mixing

In GDPP EUV source devices, glancing incidence mirrors (GIM) are applied. These can consist of thin films of high EUV reflectivity materials (e.g. $\mathrm{Ru}, \mathrm{Mo}, \mathrm{Pd}$, etc...). Understanding erosion mechanisms as well as additional thermodynamic and radiation-induced mechanisms is critical in the design of GIM materials for high-volume manufacturing (HVM) operation. In addition, diagnosis of environmental conditions, mirror material surfaces are exposed to, is critical to the advancement of GIM design. However, any materials improvement will have to be coupled with debris mitigation schemes near the source due to the high heat load electrodes at the source will be exposed to under HVM operation. In addition, understanding and diagnosis of the condenser optics region (COR) will be important to determine which erosion mechanisms will dominate at the surface. For example, off-band radiation can lead to local heating of the GIM surface. Therefore the effect of fast ion/neutrals at the GIM surface must be accounted 
for under these conditions. This paper summarizes key particle/surface mechanisms important for the design of GIM surfaces for HVM GDPP EUV devices.

\section{Plasma-surface interactions in DPP-EUV source electrode and condenser optic materials}

In GDPP EUV devices electrodes at the source are exposed to short (10-20 nsec) high-intensity plasmas leading to a variety of erosion mechanisms. Erosion of the electrodes is dictated by the dynamics of the plasma pinch for configurations such as: dense plasma focus, Z-pinch and capillary. The transient discharge deposits $1-2 \mathrm{~J} / \mathrm{cm}^{2}$ per pulse on electrode surfaces. Large heat flux is deposited at corners and edges leading to enhanced erosion. Understanding of how particular materials respond to these conditions is part of rigorous design of GDPP electrode systems. Erosion mechanisms can include: physical sputtering, current-induced macroscopic erosion, melt formation, droplet and particulate ejection. Erosion at the surface is also governed by the dynamics of how plasma can generate vapor cloud leading to a self-shielding effect, which results in ultimate protection of the surface bombarded. Determining whether microscopic erosion mechanisms such as: physical sputtering or macroscopic mechanisms such as melt formation and droplet ejection remains an open question in GDPP electrode design. This is because such mechanisms are inherently dependent on the pinch dynamics and operation of the source.

Critical to the performance of future EUV source systems is how operation of the source affects the region near and around the condenser one (C1) optics, namely: collector optics. Degradation of the condenser optics is presently one key challenge for existing and future EUV source systems that will need to perform at frequencies greater than $5 \mathrm{kHz}$ to reach powers near and above $100 \mathrm{~W}$ at IF meeting HVM demands. Operation under these conditions will lead to heavy electrode erosion and surface contamination, and even with debris mitigation schemes, condenser optics will face nontrivial degradation problems. Degradation of collector optics includes: EUV-induced processes (e.g., oxidation, carbonization), erosion and deposition of debris from various components in EUVL devices. Materials solutions will not likely lead to a factor 10-100 improvement in EUVL source performance. However, it is the understanding of C1 optics response to the operation of the source and debris mitigation schemes that can lead to a "window of opportunity" with integrated solutions on $\mathrm{C} 1$ optics that ultimately result in significant improvements to EUVL source performance.

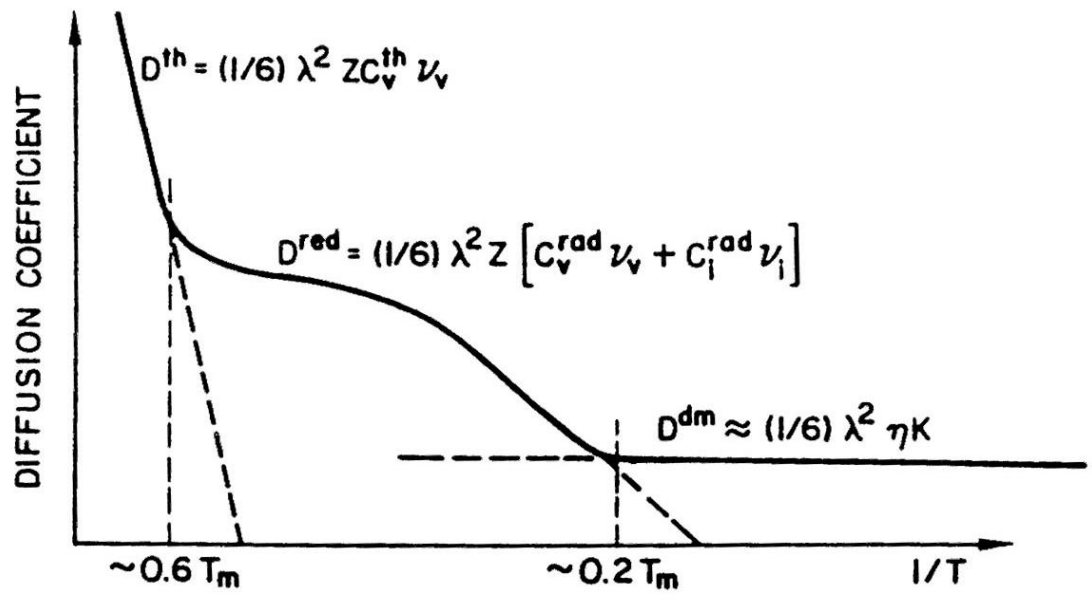

Figure 1: Schematic plot of the relative importance and temperature dependence of displacement mixing, radiation-enhanced and thermally-activated mechanisms (e.g., Gibbsian segregation).

In order to address these issues one must complement debris mitigation efforts with a thorough directed fundamental and pragmatic study of the behavior of condenser optic material surfaces under high-power EUV source conditions. This can only be done by careful diagnosis of the condenser optic region (COR) in existing EUV source devices. Fundamental understanding of the impact EUV radiation and additional particle interactions have on plasma-facing condenser mirrors can lead to the design of advanced collector optic materials that possess unique surface properties 
extending CO (condenser optics) lifetime and enhancing EUV source performance. In GDPP devices, generation of EUV light is collected by GIM, as stated earlier. The GIM is the first condenser optic of about 6-7 condenser optical systems in the EUVL device. Synergy in environmental conditions can have significant effects on GIM performance. For example, how singly-charged fast ions and neutrals created at the source induce surface damage of candidate optical materials interact with off-band radiation heating of the GIM surface. Such synergistic effects can be studied with experimental facilities such as IMPACT (Interaction of Materials with charged Particles And Components Testing) at the Argonne National Laboratory.

Conceptually, the phenomenon of bombardment-induced compositional changes is simplest when only athermal processes exist such as: preferential sputtering (PS) and collisional mixing (CM). Preferential sputtering occurs in most MCS due to differences in binding energy and kinematic energy transfer to component atoms near the surface. Collisional mixing of elements in MCS is induced by displacement cascades generated in the MCS by bombarding particles/clusters and is described by diffusion model

Irradiation can accelerate thermodynamic mechanisms such as Gibbsian adsorption or segregation (GA) leading to substantial changes near the surface with spatial scales of the order of the sputter depth (few monolayers). GA occurs due to thermally-activated segregation of alloying elements to surfaces and interfaces reducing the free energy of the alloy system. Typically, GA will compete with PS and thus, in the absence of other mechanisms, the surface reaches a steady-state concentration approaching that of the bulk. However when other mechanisms are active, synergistic effects can once again alter the near-surface layer and complex compositions are achieved.

These additional mechanisms include: radiation-enhanced diffusion (RED) due to the thermal motion of nonequilibrium point defects produced by bombarding particles near the surface, radiation-induced segregation (RIS), a result of point-defect fluxes, which at sufficiently high temperatures couples defects with a particular alloying element leading to compositional redistribution in irradiated alloys both in the bulk and near-surface regions. Figure 1 shows the temperature regime where these mechanisms are dominant. All of these mechanisms must be taken under account in the design of proposed advanced materials in addition to considering other bombardment-induced conditions (i.e., clusters, HCI, neutrals, redeposited particles, debris, etc...).

\section{EXPERIMENTAL SETUP}

\subsection{IMPACT (Interaction of Materials with charged Particles And Components Testing)}

IMPACT is a new experiment in the PRIME facility at ANL and is shown schematically in Fig. 2 with a photograph in the inset. IMPACT is the first component of PRIME recently built and currently running for the measurement and testing of charged-particle interactions with multi-component systems. IMPACT consists of a well-collimated ion source with an energy range between 50 and $5000 \mathrm{eV}$, fluxes of $10^{11}-10^{17}$ ions $/ \mathrm{cm}^{2} / \mathrm{s}$ and incident angles from normal incidence to about 60-70 degrees with respect to normal. Base pressures are attainable down to $10^{-10}$ Torr with gas inlets for controlled impurity desorption/adsorption experiments. In addition, an in-situ heating design can vary the sample temperature from ambient conditions to about $100{ }^{\circ} \mathrm{C}$. IMPACT currently has a quartz crystal microbalance dual crystal unit (QCM-DCU) diagnostic system for in-situ real-time total erosion measurements and will soon have a low-energy ion scattering spectroscopy (LEISS) system for in-situ multi-component erosion measurements. The sample is fixed on a rotatable manipulator, whose rotation axis lies in the plane of the sample surface. This allows for angle-ofincidence measurements of the irradiated surface. In addition, the sample holder includes an in-situ UHV heater system with thermocouple located underneath the sample for temperature variation tests. The heater is equipped with a setpoint controller with the capability of also setting the heating rate $\left({ }^{\circ} \mathrm{C} / \mathrm{sec}\right)$. This is important since various microstructures and surface morphologies are obtained based on the local surface condition. The sample sits in a stainless steel cup allowing for exchange of samples in vacuo with a manipulator and transfer lock system. When the sample is removed from the ion beam, the beam is collected by a faraday cup for beam profile analysis. The faraday cup consists of four small pinholes about $0.125 \mathrm{~mm}$ in diameter separated $2 \mathrm{~mm}$ apart. A Labview code controls the raster unit of the ion source and scans two directions ( $\mathrm{x}$ and $\mathrm{y}$ ) perpendicular to the beam. This allows for a beam profile to be generated as shown in Figs. 3 and 4. This particular profile was done for a $\mathrm{Xe}^{+}$beam at $1 \mathrm{keV}$ with a current of approximately $2 \mu$ A giving a flux of about $10^{15} \mathrm{ions} / \mathrm{cm}^{2} / \mathrm{sec}$. 


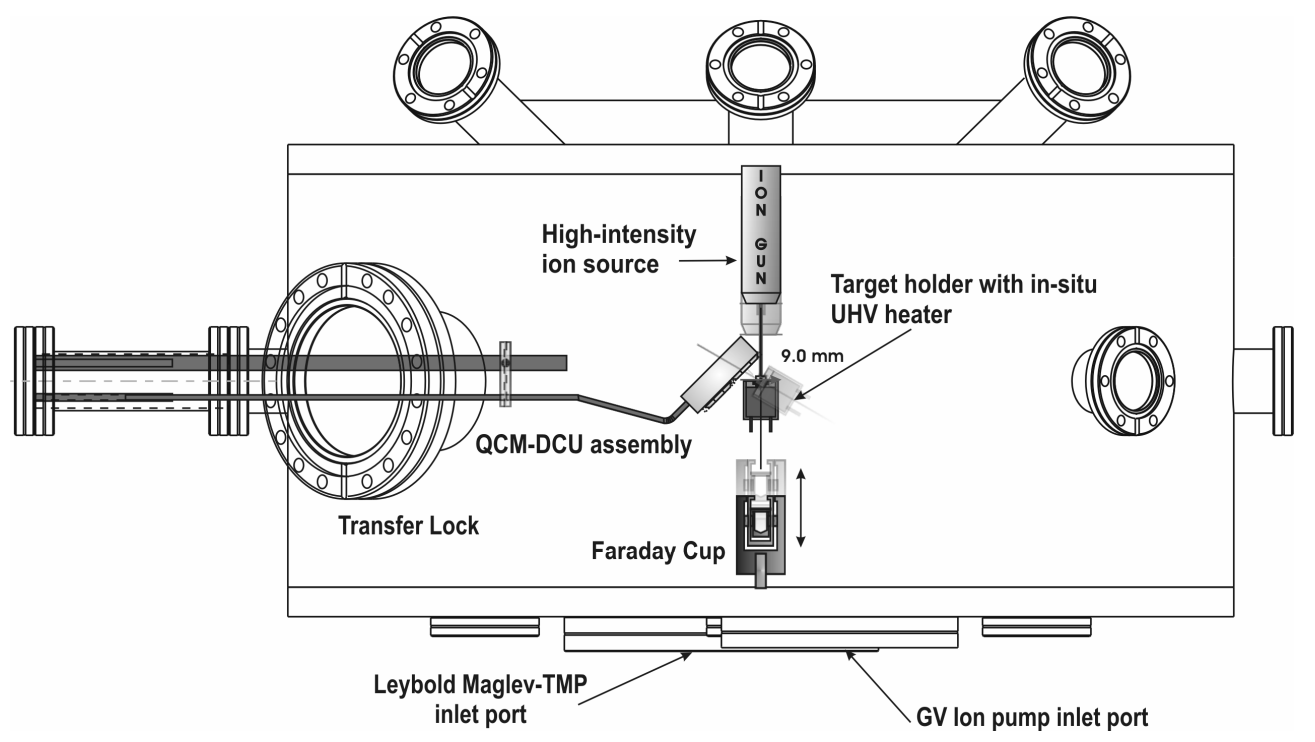

Figure 2: The quartz crystal microbalance - dual crystal unit (QCM-DCU) system in the IMPACT (Interaction of Materials with charged Particles And Components Testing) experiment at the Argonne National Laboratory.

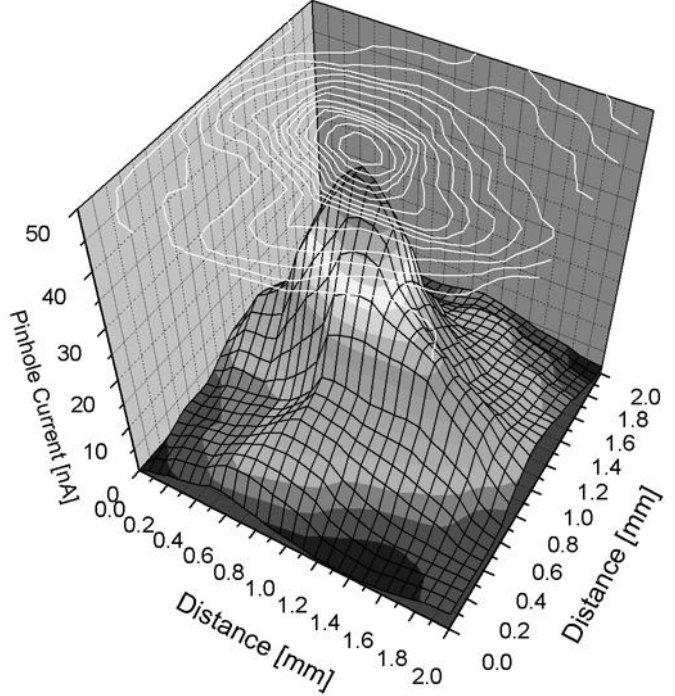

Figure 3: Xenon ion beam profile measured by in-situ Faraday cup placed along the ion-beam axis centered below the IMPACT sample holder.

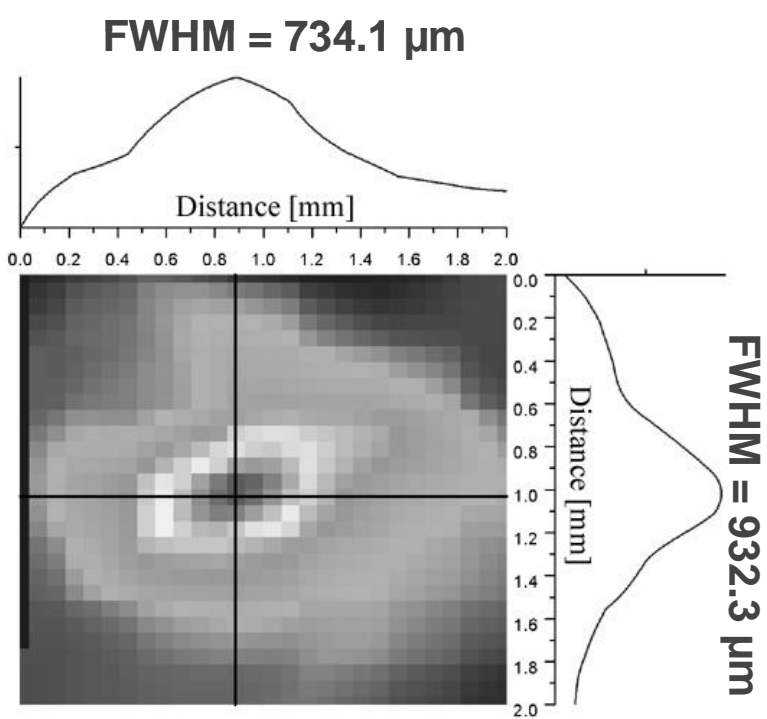

Figure 4: Xenon ion beam profile $\mathrm{M}^{2}$ image measured by Faraday cup. FWHM of ion beam corresponds to $1 \mathrm{keV}$ Xe ions with about $2 \mu \mathrm{A}$ total current equating to an incident ion flux of about $2 \times 10^{15}$ ions $/ \mathrm{cm}^{2} / \mathrm{sec}$.

Future additions to IMPACT will include diagnostics for measurement of surface evolution (roughening, smoothing, multi-layer or GIM structure), auger emission spectroscopy (AES) with an in-situ electron gun and low-energy ion scattering spectroscopy (LEISS) as mentioned above. LEISS works on the principles of conservation of energy and momentum. An incident charged particle incident on a multi-component surface will backscatter with an energy characteristic of the mass of the target components from which it's scattered. Therefore, the energy spectrum obtained is the mass spectrum of the surface atoms. The resolution of this technique is based on neutralization mechanisms that result in backscattered ions containing information only of the surface. 
In-situ diagnosis is important for several reasons. A surface under ion irradiation is actively changing during the exposure dose. For example, radiation-induced segregation will drive certain target components to the surface, while radiation-enhanced diffusion will drive them away from the surface. Adsorbates will be actively on a given surface dictated by the surface kinetics and thermodynamics and their role on mechanisms such as sputtering and reflection can only be assessed by actively interrogating the surface irradiated.

Accelerated testing of candidate GIM or ML can be done due to the large range of flux of ion-bombardment and longterm electron-gun exposure tests. IMPACT also is equipped with an in-situ UHV heater providing for thermal annealing tests while the sample's surface is diagnosed and simulating off-band radiation heating effects. This paper presents initial experiments in IMPACT of total single-component erosion by use of the QCM-DCU diagnostic system.

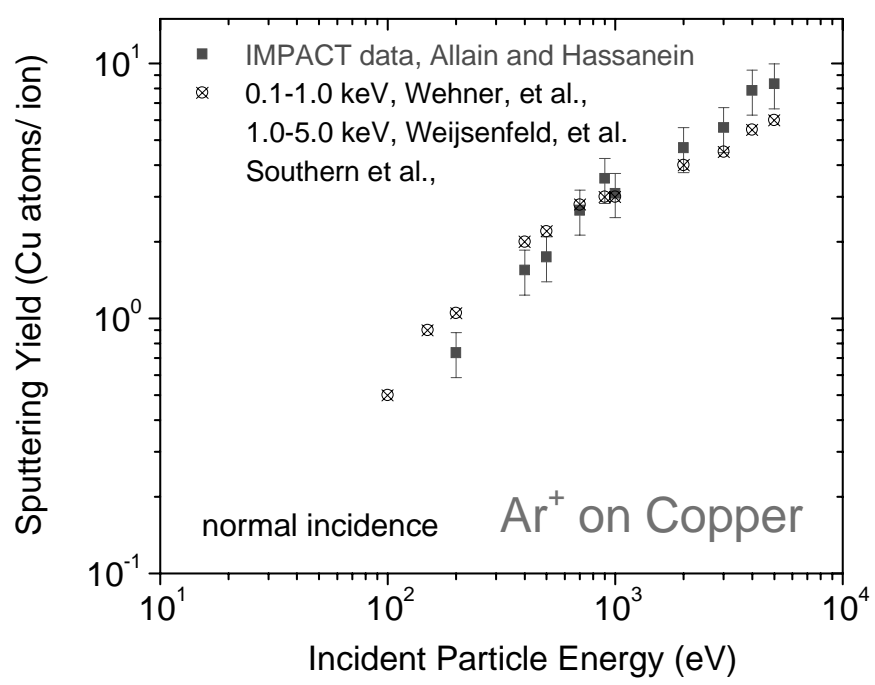

Figure 5: Sputtering yield of copper bombarded by singly-charged argon at normal incidence in the IMPACT (Interaction of Materials with charged Particles And Components Testing) experiment at the Argonne National Laboratory.

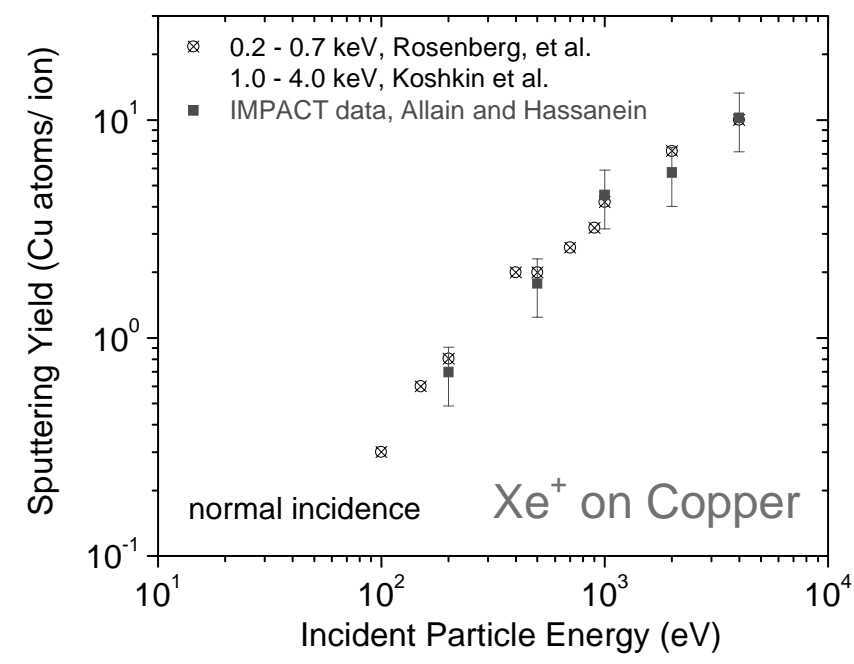

Figure 6: Sputtering yield of copper bombarded by singly-charged xenon at normal incidence in the IMPACT (Interaction of Materials with charged Particles And Components Testing) experiment at the Argonne National Laboratory. 


\subsection{Data analysis with a QCM-DCU system}

The quartz crystal microbalance technique is a mature and well-developed diagnostic metrology tool measuring mass loss from materials irradiated by energetic particles. One particular version of this technique is the use of a quartz crystal microbalance - dual crystal unit system capable of measuring monolayer-level erosion of surfaces irradiated by charged-particle bombardment. This particular version uses one crystal oscillator to measure deposition from an eroding surface, while the second measures the background ambient simultaneously. This also contends with the variability of the oscillators with background system pressure and long-temporal, temperature-dependent effects. Background ambient changes can be picked up by the ultra-sensitive oscillator and thus when measuring monolayerlevel deposition over a relatively small dose, the use of a deposition and reference oscillator system can improve measurement accuracy.

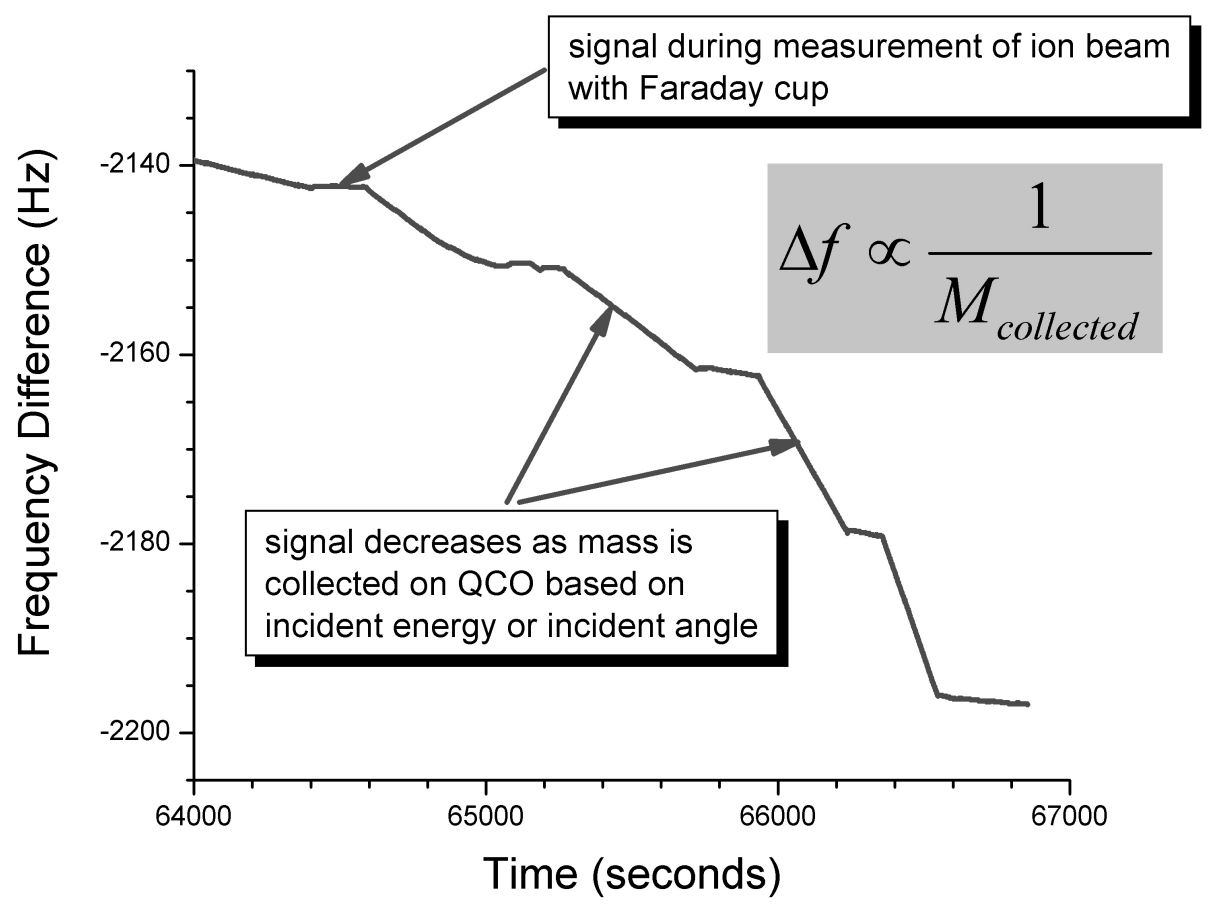

Figure 7: Frequency difference results using the quartz crystal microbalance - dual crystal unit (QCM-DCU) system in the IMPACT (Interaction of Materials with charged Particles And Components Testing) experiment at the Argonne National Laboratory. The data shows a decrease in the frequency difference corresponding on a mass increase on the collector crystal of the QCM-DCU system. Each slope corresponds to either a particular incident particle energy or angle of incidence. The near-flat regions of the signal are background data while the sample is removed from the ion beam and the Faraday cup is placed to read the net current to the sample.

Figures 5 and 6 show results for total sputtering measurements for $\mathrm{Ar}^{+}$and $\mathrm{Xe}^{+}$bombardment at normal incidence for energies ranging from $200-5000 \mathrm{eV}$. The results are compared to well-known experimental data, thus serving as a good calibration technique for the setup in IMPACT. The QCM-DCU technique works by depositing an amount of eroded material on a gold-covered quartz crystal oscillator. The oscillator vibrates at a fundamental frequency of about $6 \mathrm{MHz}$. As mass is deposited on the surface of the oscillator, it's frequency of the oscillator decreases. The measurement of total physical sputtering is very sensitive therefore a dual-crystal unit is applied. The unit works by one oscillator monitoring eroded material and the other background frequency. The frequency difference is therefore utilized in the calculation of the sputtering yield. Care is taken to several factors: the sticking coefficient of known species to the collector surface of known surface composition, the collecting solid angle and the resputtering of collected material by fast, reflected ions or neutrals. Surface compositions are assumed based on previous surface analysis results. Fig. 7 shows raw frequency data plotted versus time of a particular run. The decreasing slope corresponds to a collection of mass on the collector crystal. The different slopes correspond to a particular incident particle energy or angle. The 
nearly flat trends correspond to the sample being retracted away from the beam for the ion beam current measurement by the Faraday cup.

\section{MODELING}

\subsection{Physical sputtering modeling with BCA-based and atomistic-based simulation codes}

Modeling of physical sputtering is well known and the field quite mature. For energies above about $100 \mathrm{eV}$, binary collision approximation (BCA) codes are often used to estimate erosion from various material surfaces. For example in Fig. 8 the sputtering from a W-Cu alloy is modeled. The sputtering yield of $100 \% \mathrm{Cu}$ from $1 \mathrm{keV} \mathrm{Xe}^{+}$bombardment coincides with the experimental result shown for $\mathrm{Cu}$ bombardment. This prediction allows for the use of ITMC on a number of studies on multi-component surface sputtering.

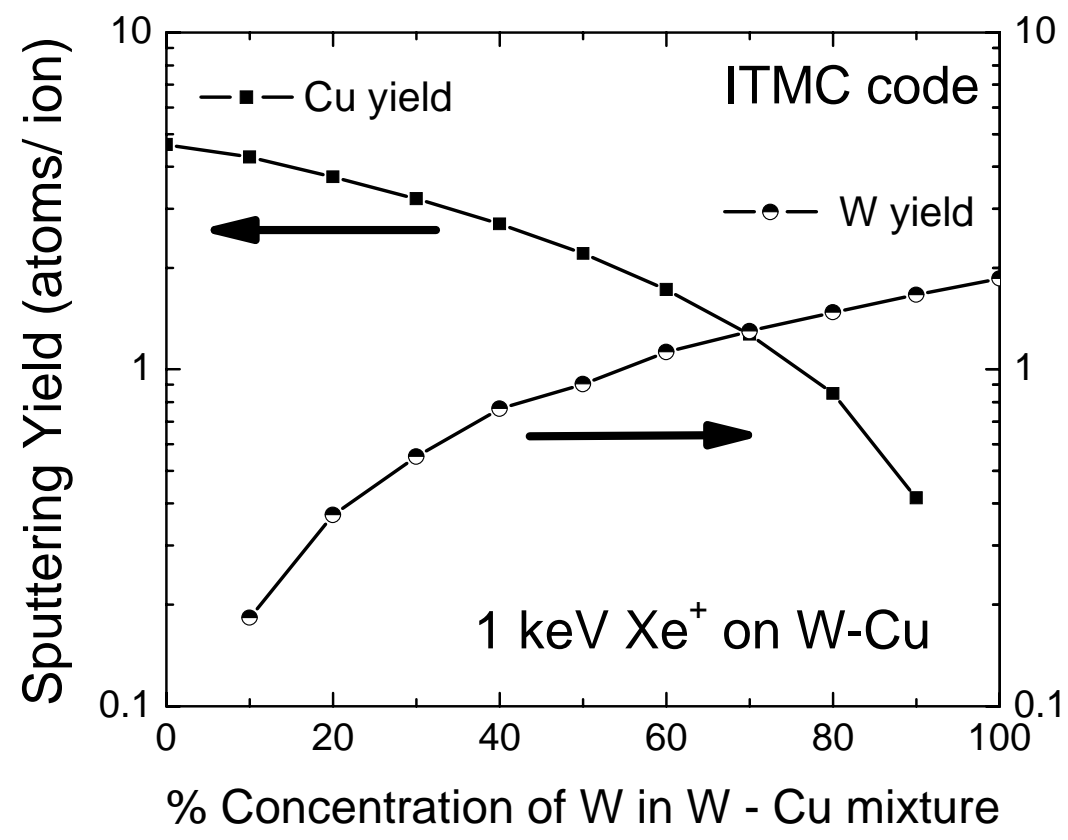

Figure 8: The Ion Transport in Materials and Compounds code developed at the Argonne National Laboratory calculates the partial sputtering yield of $\mathrm{Cu}$ and $\mathrm{W}$ from a W-Cu mixed material bombarded by $1 \mathrm{keV}$ Xe ions at normal incidence. This system is used as a pseudo-alloy with properties able to withstand large heat fluxes in EUV source devices.

\subsection{Atomistic modeling of $\mathrm{HCI}$ interaction with targets}

Due to the short-lived plasma pinch generated in GDPP devices a certain flux of highly-charged ions (HCI) will arrive at the mirror surface. HCI have very high potential energy gained by the ion during the multiple ionization events. For example, a Xe ${ }^{+10}$ highly-charged ion has of about $1 \mathrm{KeV}$ of potential energy as shown in Fig. 9. When these HCI collide with a surface, it almost instantly captures electrons from the surface by a resonant capturing process and forms a Hollow Atom (HA). The electrons in the HA are located at Rydberg (hydrogen-like) orbitals with large radii. The resonant capture of surface electrons leads to a significant surface charging effect.

The consequences of local charge accumulation to the target vary depending on the conductivity of the free electron concentration of the target material. If the target is an insulator or a semiconductor, the charged area could undergo a "Coulomb explosion" (CE) caused by a strong Coulomb repulsion between the positively ionized target atoms. According to this model, the surface of the sample starts to blow up thus producing significant damage to the surface and a high sputtering yield, made up of secondary ions as well as surface neutral atoms. 


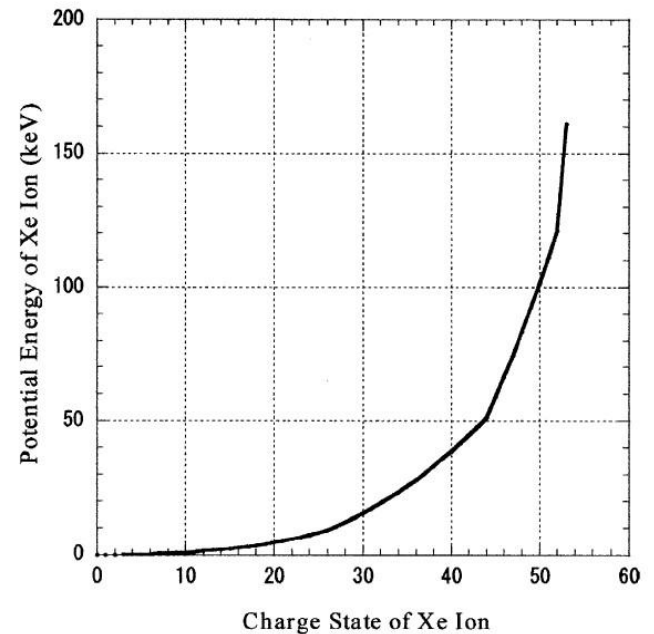

Figure 9: Potential energy of highly-charged $\mathrm{Xe}^{+\mathrm{q}}$ ions.
Sputtering processes of Silicon in the bombardment of highly charged ions (HCIs) are studied using Molecular Dynamics simulation based on a simple model without the neutralization effect. Assuming the potential energy of the HCI transferred to target is stored as the electrostatic energy of $\mathrm{Si}$ atoms ionized by the $\mathrm{HCI}$, the Si ions up to 375 are embedded on a Si (100) surface as an initial condition, resulting in Coulomb explosion. The dynamics of particle ejection (sputtering) from the surface and crater formation on the surface are simulated. The formation and propagation of the shock wave and rapid increase of the sputtering yield are seen during relaxation process.

MD models of the hollow atom formation, surface charge neutralization and surface damage caused by kinetic and potential energy have been designed and are currently modeling additional $\mathrm{HCI} /$ surface interactions.

\section{RESULTS AND DISCUSSION}

\subsection{Selection of electrode materials in DPP EUV devices}

Selection of materials for GDPP electrodes depends on the microstructure desired to minimize erosion and maximize thermal conductivity. Three materials have been identified and the sections manufactured with metallographic analysis and microanalysis performed. The powder composite materials inherited the structural characteristics of the initial powders, determined by the processes of combined restoration of tungsten and nickel oxides $\left(\mathrm{WO}_{3}\right.$ and $\mathrm{NiO}$ from $\mathrm{NiCO}_{3}$, for instance) and copper molybdat $\left(\mathrm{MoCuO}_{4}\right)$. Dry hydrogen (the dew point temperature is above $20{ }^{\circ} \mathrm{C}$ ) facilitates the formation of the heterogeneous conglomerates in W-Ni-powders, which do not collapse at sintering or saturate the material (Fig. 10), and spheroidizing of molybdenum particles and re-crystallization through the liquid phase in the conditions of sintering the composite consisting of molybdenum and copper (Fig. 11). For comparison, the structure is shown in Fig. 12 obtained from tested W-Ni powders. The structure of the materials was studied by means of scanning electron microscopy (SEM) of the secondary electrons.

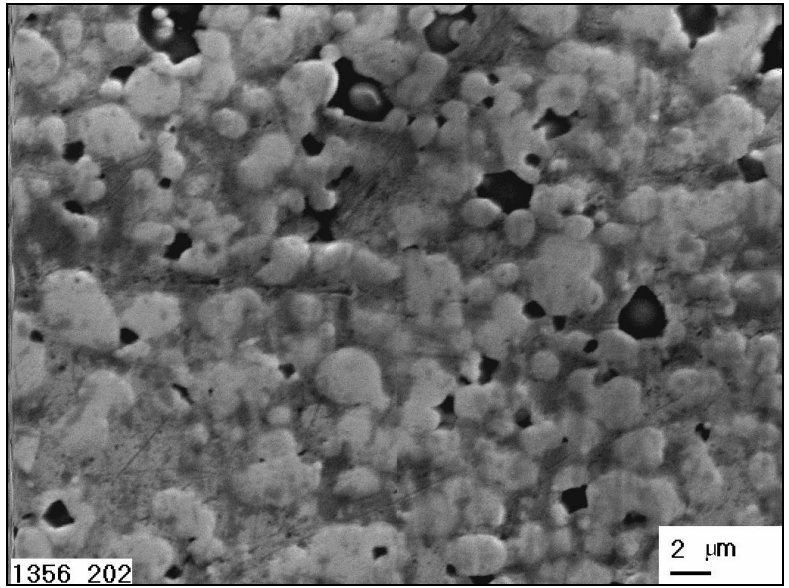

Figure 10: The structure of the $\mathrm{W}-\mathrm{Cu}-\mathrm{Ni}-\mathrm{LaB}_{6}$ pseudo alloy $(\times 540)$.

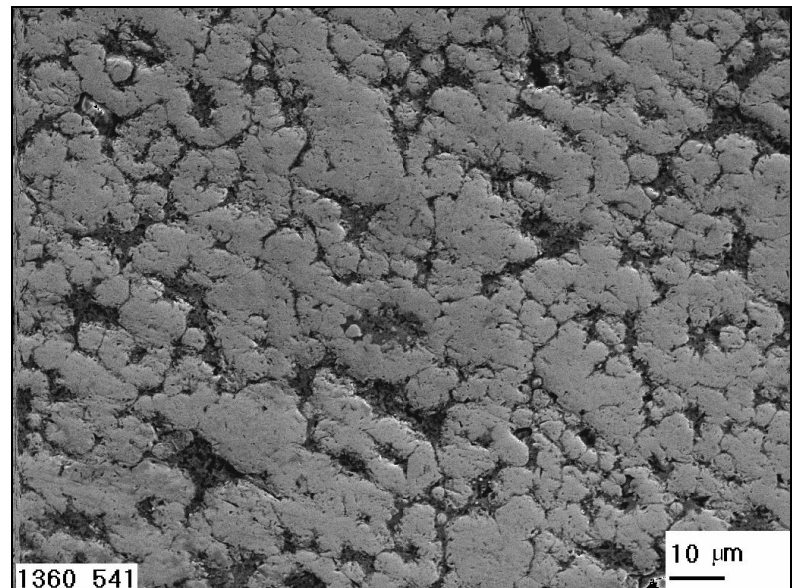

Figure 11: The structure of the $\mathrm{Cu}-44 \% \mathrm{Mo}-1 \% \mathrm{LaB}_{6}$ pseudo alloy $(\times 2000)$ 
So, one can speculate that the hydrogen observed by the ionic spectroscopy after exposing the samples may be caused by that environment, in which the powders are manufactured, sintered, and additionally annealed. In regards to the beryllium observed on the anode surface after exposure to the xenon plasma, one may suppose two possible explanations, each of which requires additional verification. The construction may contain beryllium bronze; or the construction may contain $\mathrm{Al}_{2} \mathrm{O}_{3}$ or $\mathrm{BeO}$ based ceramics. Both cases may be the reason for enrichment of the surface samples by these elements during the heating phases.

For systems with the absence of the component interactions, the arc xenon plasma impact to the electrode materials does not cause a noticeable change of durability: for $\mathrm{MoCuLaB}_{6}: \mathrm{HV}=1600-1690 \mathrm{MPa}$; and for $\mathrm{Cu}-\mathrm{Al}_{2} \mathrm{O}_{3}: \mathrm{HV}=660 \mathrm{MPa}$ through the whole height of the anode. In the tungsten and copper based composites, when presence of nickel exists, the mutual dissolution of the elements is increased ( $\mathrm{W}$ is dissolved in $\mathrm{Cu}-\mathrm{Ni}$ melt, for instance). At cooling, it may be accompanied by either forming non-equilibrium solid solution, or solidification; which is conformed by the increasing the firmness of the upper part of the anode (3380 MPa compared to $3020 \mathrm{MPa}$ in its lower part).

To provide more careful analysis, one should investigate the dependence of electro-conductive composites on heat resistance subject to arc discharges of powerful heat fluxes (up to $10^{7} \mathrm{~W} / \mathrm{m}^{2}$ ). We also plan to continue analyzing the propagation of cracks, observed on the surface layer of the anode material and deep into the bulk. For that, the precise method of manufacturing is required for further insight on crack development and propagation.

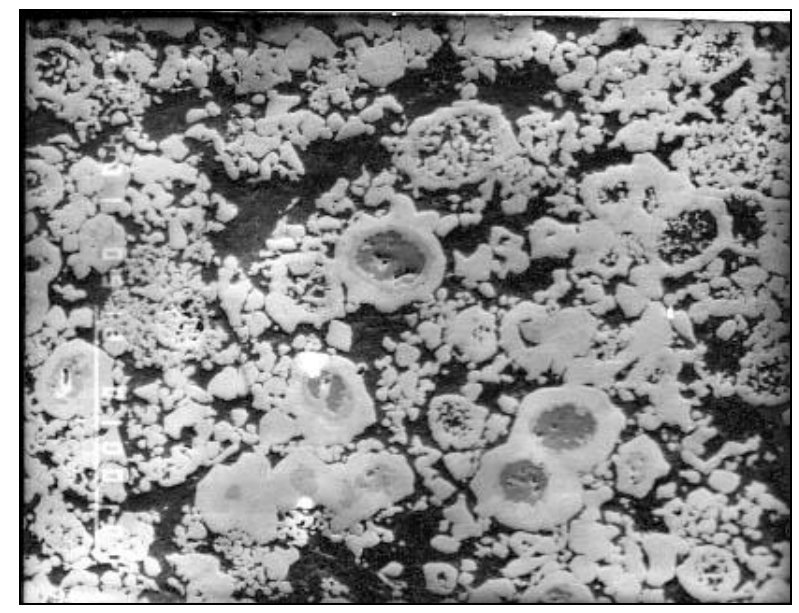

Figure 12: The structure of $\mathrm{W}-\mathrm{Cu}-\mathrm{Ni}$ pseudo alloy, produced by class $\mathrm{W}-\mathrm{Ni}$ powder $(\times 400)$.

\subsection{Oblique incidence erosion of ruthenium bombarded by $\mathrm{Xe}^{+}$}

The physical sputtering yield of $\mathrm{Xe}^{+}$bombardment of ruthenium at 45-degrees was measured in IMPACT. The incident particle energy ranged from 500-1000 eV. The sputtering yield rises from about $0.1 \mathrm{Ru}$ atoms/ ion at $500 \mathrm{eV}$ to about $0.4 \mathrm{Ru}$ atoms/ ion for $1500 \mathrm{eV}$. $\mathrm{Xe}^{+}$bombardment shows no indication of non-linear erosion. This is because the BCAbased code, ITMC is used to model the system for a number of incident particle energies. The model implemented in the ITMC code is consistent with the linear sputtering model. There is very good agreement between the model and experimental data and thus do not expect for the Ru yield to rise non-linearly with temperature. For GIM the measurement of oblique incidence of fast ions (e.g. $\mathrm{Xe}^{+}$) is of interest. This is due to the solid angle collecting EUV light providing for very oblique angles of incidence to fast singly-charged ions. Multiply-charged ions must also be accounted for as stated earlier due to the level of potential energy these carry to the ruthenium surface. In these measurements a ruthenium oxide layer was assumed to deposit on the collecting crystal of the QCM-DCU diagnostic. This needs further investigation using post-exposure surface analysis. 


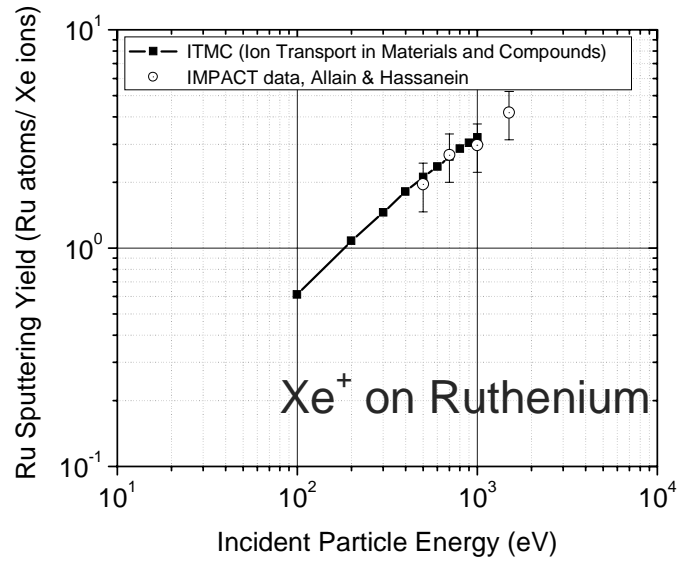

Figure 15: SEM image of tungsten surface after 15 exposures to xenon plasma stream.

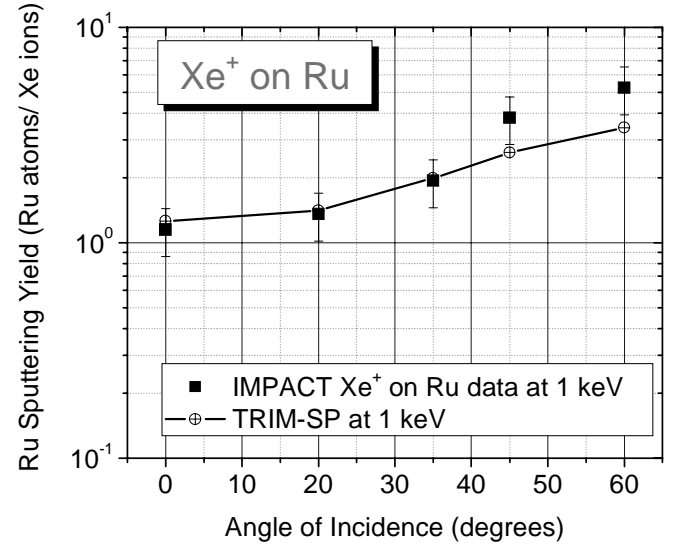

Figure 16: SEM image of tungsten surface after 15 exposures to hydrogen plasma stream.

\section{CONCLUSIONS}

Plasma-facing EUV source device materials will need to be carefully designed to handle the harsh environment they will be exposed to. Electrode materials must be designed to provide erosion resistance and good thermal conductivity. High-density plasmas in GDPP devices will lead to serious erosion challenges as HVM conditions are attained. However, with proper electrode design and robust debris mitigation schemes, some opportunity exists for operation in HVM. Condenser mirror optics faces the same challenge. The mirror surface will have to be designed to contend with incident debris, highly-energetic singly-charged and multi-charged particles and fast neutrals. More importantly, the synergy of such fluxes combined with background impurities and off-band radiation will ultimately dictate the fate of plasma-facing mirrors. Therefore, proper design coupled to debris mitigation schemes will enable emerging lithography technologies to operate under HVM conditions. This goal can be presently attained by designing well-diagnosed, controlled off-line experiments to understand synergistic erosion mechanisms that can lead to advanced mirror designs.

\section{ACKNOWLEDGEMENTS}

The authors would like to acknowledge the help of Perry Plotkin, Dan Rokusek, Hussain Nomanbhai, Michael Hull, and Chris Chrobak.

\section{REFERENCES}

1. S. Bajt, H.N. Chapman, N. Nguyen, J. Alameda, et al. "Design and Performance of capping layers for extremeultraviolet multilayer mirrors", Applied Optics, Vol. 42, No. 28, 2003.

2. J.P. Allain, M.D. Coventry, D.N. Ruzic, "Temperature dependence of lithium sputtering under low-energy singlycharged particle bombardment", to be submitted in Journal of Applied Physics, 2004.

3. Terasawa, M., Z.A. Insepov, T. Sekioka, A.A. Valuev, and T. Mitamura. "Sputtering Due to Coulomb Explosion in Highly Charge Ion Bombardment." Nuclear Instruments \& Methods in Physics Research B 212 (2003) 436-41.

4. J.P. Allain, "QCM-DCU diagnostic system for the measurement of physical sputtering of liquid-metals", to be submitted in Review of Scientific Instruments, 2004. 